\title{
Synthesis of carbon nanostructures using microwave enhanced chemical vapor deposition and its potential application to ammonia sensing
}

\author{
Kure Nicodemus ${ }^{1,2}$, Yunusa Zainab ${ }^{3,4}$, Hamidon M Nizar ${ }^{1,3}$, Daniel H Isaac ${ }^{2}$, Ibrahim \\ I Lakin ${ }^{2}$ \\ ${ }^{1}$ Department of Electrical and Electronics Engineering, Universiti Putra Malaysia, Selangor, Malaysia. \\ ${ }^{2}$ Department of Physics, Kaduna State University, Kaduna, Nigeria. \\ ${ }^{3}$ Institute of Advanced Technology, Universiti Putra Malaysia, Selangor, Malaysia \\ ${ }^{4}$ Department of Electrical Engineering, Bayero University, Kano, Nigeria.
}

Corresponding E-mail: kurenicodemus@gmail.com

Received 02-06-2021

Accepted for publication 21-07-2021

Published 23-07-2021

\begin{abstract}
In this paper, Carbon Nanostructures (CNS) were directly synthesized on gallium orthophosphate (GaPO4). An alternative microwave enhanced chemical vapor deposition (MECVD) technique was used to synthesized the CNS via commercial microwave oven with operating power of $600 \mathrm{~W}$ at $2.45 \mathrm{GHz}$. Microwave heating provides the temperature for catalytic decomposition of polyethylene at $750{ }^{\circ} \mathrm{C}$ for 4 minutes under atmosperic pressure of 0.81 mbar. Characterization of the as-grown CNS was carried out using Raman spectroscopy and Field Emission Scanning electron microscope (FESEM). Raman spectroscopic investigation reveals the CNS quality of 0.92 and the field emission Scanning electron microscope (FESEM) analysis shows twisted hollow-like CNS structures. The material was deployed as a sensor without any post treatment so as to investigate its potential application in the sensor industry. Different concentrations of ammonia (NH3) gas from $0.06 \%$ to $1 \%$ were exposed.
\end{abstract}

Keywords: Carbon nanostructures; Raman; Ammonia sensing; Microwave oven; Chemical vapor deposition

\section{INTRODUCTION}

A mmonia $\left(\mathrm{NH}_{3}\right)$ one of the most highly toxic gas, environmental pollutants and yet being utilized in many food processing, medical diagnosis and chemical industries. Any leak in these systems may cause health hazards $[1,2]$. Hence, detection of $\mathrm{NH}_{3}$ in trace amount is very crucial in terms of health and environmental sectors. Therefore, a reliable, sensitive and selective gas sensor is required to detect low traces of concentration of $\mathrm{NH}_{3}$ gas. In recent years, novel materials such as carbon based nanostructures are exploited for $\mathrm{NH}_{3}$ gas detection which varies in their sensing mechanism and concentration range due to its exceptional properties amongst such are high thermal and mechanical stability, high surface-area-to-volume ratio, good metallic and semi conducting behaviour [1, 2, 3, 4]. Carbon nanostructures have been synthesized directly on gallium orthophosphate using alcohol catalytic chemical vapor deposition (ACCVD) as reported by [5]. In this paper, the authors proposed to use microwave enhanced chemical vapor deposition to synthesize CNS directly on $\mathrm{GaPO}_{4}$ and explore its potential application as an ammonia gas sensor. Gallium orthophosphate $\left(\mathrm{GaPO}_{4}\right)$ is piezoelectric in nature

Researchers embarked in utilizing microwave heating 
compared to conventional heating techniques due to its rapid, fast and volumetric heating [6,7,8]. Microwaves are kind of electromagnetic radiations. The microwave synthesis of carbon nanostructures (CNS) attract attention compared to arc discharge [9], laser ablation [10], chemical vapor deposition [11] due to its advantages. This work, attempt to synthesis CNS from plasma catalytic decomposition of polyethylene on gallium orthophosphate via commercial microwave oven, which has less time consumption. Characterization of the synthesized CNS was carried out using Raman Spectroscopy, Field Emission Scanning Electron Microscope (FESEM) and subsequently gas sensing measurements were conducted.

\section{MATERIALS AND METHODS}

\section{A. Synthesis Procedure}

All chemicals are used as procured without further purification. Polyethylene $(100 \mathrm{mg})$ was used as carbon source; iron (III) nitrate was impregnated on gallium orthophosphate $\left(\mathrm{GaPO}_{4}\right)$ as catalyst and substrate. The same $\mathrm{GaPO}_{4}$ substrate was also employed as already published in $[1,2,5] . \mathrm{GaPO}_{4}$ was found to have high thermal stability enhances the direct synthesis of carbon nanostructures [5]. The impregnated substrate was placed in oven at $200{ }^{\circ} \mathrm{C}$ for 1 hour for the catalyst to reduce to iron oxide and interact with the gallium orthophosphate [12]. Commercial microwave oven was modified to insert cylindrical quartz tube which serves as the reaction chamber. Carbon source and substrate was placed inside the close chamber. Rotary vacuum pump was used to reduce the atmospheric pressure of the chamber to $0.81 \mathrm{mbar}$. The microwave oven was switch on to the start the synthesis process. The generated plasma provides the temperature needed for catalytic decomposition of polyethylene, which changes color from white to yellow. Thermocouple was embedded inside the chamber to measure the process temperature, which was found to be at $750{ }^{\circ} \mathrm{C}$ for 4 minutes. The as grown product was allowed to reach ambient condition before characterization.

\section{B. Characterization}

Characterization analyses were carried out after the sample was collected at ambient condition. The carbon quality of the CNS was analysed using Confocal Raman spectroscopy Witec Alpha 300R with laser excitation energy of wavelength 532 $\mathrm{nm}$. Structure and morphology analysis of the CNS was carried out using field emission scanning electron microscope (FESEM, S3400N Hitachi).

\section{Gas Sensing}

An alumina substrate $1 \times 1 \mathrm{~cm}^{2}$ was used for the fabrication of the template support to mount the sensing layer using the screen printing technique. After synthesis of the sensing material, it was then integrated onto the alumina substrate. The as grown CNS acts as the sensing material and then made into a sensor placed on the platinum pads with the help of the conductive silver paste. Two gold wires were soldered on the soldered to the platinum pads. The sensor was then introduced into the gas chamber. Resistance changes were monitored by the use of Flukeview meter which was datalogged into a computer. The experimental set-up consists of two Aalborg mass flow controllers which are fed with air and ammonia gases respectively. These are connected to a command module which is connected to a computer and controlled using LabVIEW program. The gas was introduced into the chamber via the inlet from the mass flow controllers. The outlet of the sensor was connected to a fluke view multimeter which is connected to the computer via the cable. The output of the sensor is data logged with the aid of Flukeview form software installed on the computer. Different ammonia concentrations were exposed ranging from $0.06 \%$ to $0.75 \%$ concentration.

\section{RESULTS AND DISCUSSIONS}

\section{A. Characterization}

Fig. 1 shows the prominent peaks in Raman spectra at $1368 \mathrm{~cm}^{-1}$ and $1586 \mathrm{~cm}^{-1}$ as defect (D) and graphite (G) mode respectively. Defect mode indicates the level of disordered carbon atoms while graphite mode indicates the level of ordered carbon atoms in the CNS, all associated to first order Raman scattering [13]. The peak at $2604 \mathrm{~cm}^{-1}$ are associated with second order Raman scattering term as $\mathrm{G}^{\prime}$ [13]. The carbon quality is determined by the intensity ratio $\left(I_{D} /_{I_{G}}\right)$ of defect $\left(I_{D}\right)$ and graphite modes $\left(I_{G}\right)$ [14]. The carbon quality was calculated to be 0.89 . The level of defect mode was high due to the presence of amorphous carbon which serves as impurity [6]. The morphological images captured in Fig. 2 by FESEM shows a twisted hollow-like structure with small diameters. From the micrograph, irregularities in the shape may be due to the high level of defect mode as shows in Fig. 1. And the small diameters and hollow structures make them suitable for gas adsorption and detection. The resistance of the CNS will change upon interaction with gas molecules [1].

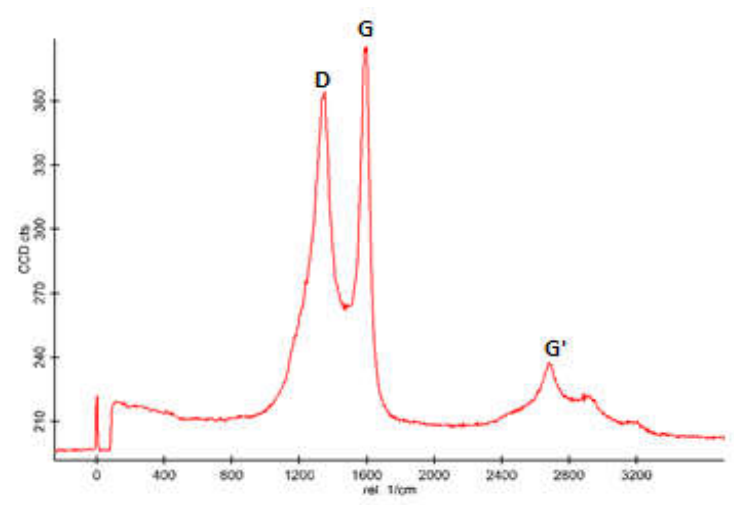

Fig. 1 Raman spectra of CNS. 


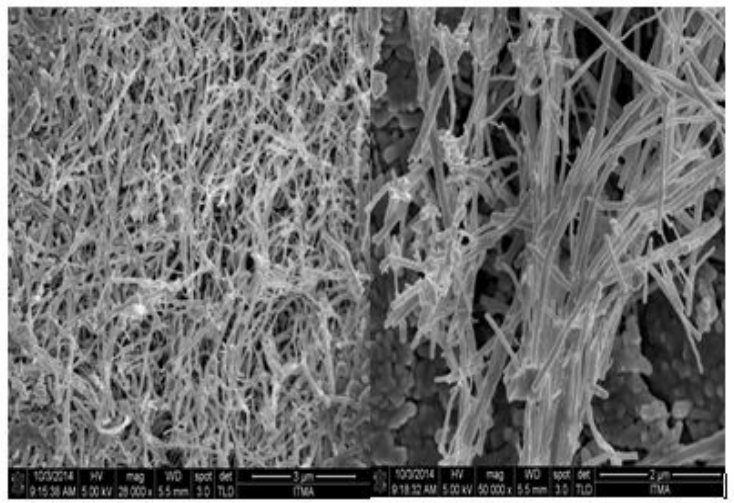

Fig. 2 FESEM micrographs of CNS at different magnification.

\section{B. Temperature Effect}

The experimental temperature process was measured using thermocouple at $750{ }^{\circ} \mathrm{C}$ as depicted in Fig. 3. Although temperature has insignificant effect, in which all the sensing was conducted at room temperature.

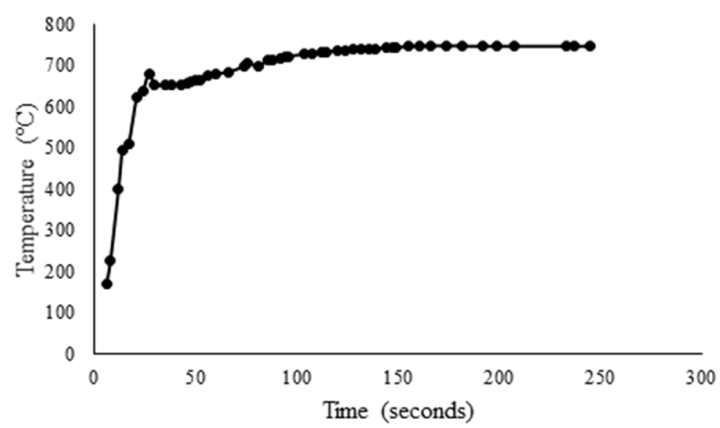

Fig. 3 Relationship between temperature and time for synthesis

\section{Gas Sensing}

The principle of sensing mechanism of CNS based gas sensor is based on change in electrical properties such as resistance which is induced by transfer of charges between gas molecules [1]. The resistance of the CNS will change upon interaction with $\mathrm{NH}_{3}$ gas molecules. Fig. 4 shows response of the CNS exposure in $\mathrm{NH}_{3}$ environment at different concentrations ranges from $0.06 \%$ to $0.75 \%$. The response behaviour shows increase in resistance during $\mathrm{NH}_{3}$ adsorption and also decrease in resistance during desorption for different ammonia concentrations from $0.06 \%$ to $0.75 \%$. The sensor response and recovery times were also observed to be good with average response time of 5 minutes and recovery time of 7 minutes respectively. Therefore, the sensing layer could be deployed for $\mathrm{NH}_{3}$ gas sensing.

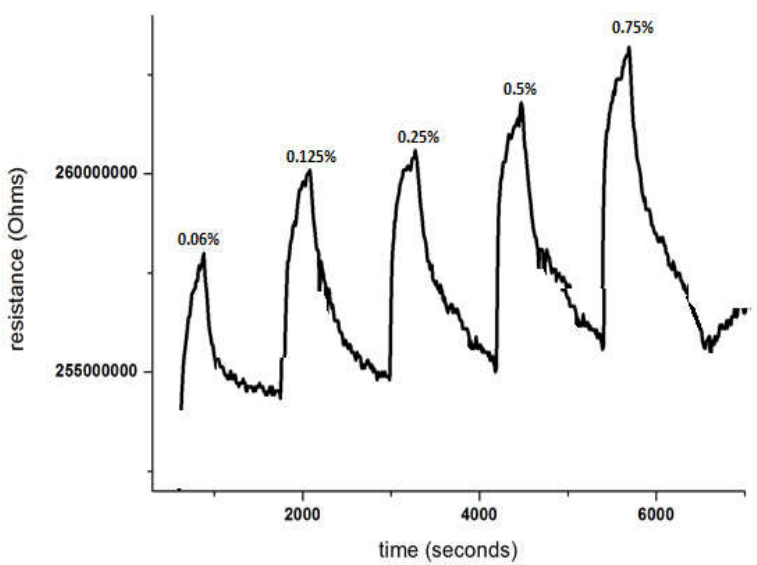

Fig. 4 Response of $\mathrm{NH}_{3}$ gas sensing concentration range of $0.06 \%$ to $0.75 \%$.

\section{CONCLUSION}

Present work shows alternative way of synthesizing CNS via microwave enhanced chemical vapor deposition on $\mathrm{GaPO}_{4}$ for 4 minutes at $750{ }^{\circ} \mathrm{C}$ under atmospheric pressure. Carbon quality was calculated to be 0.92 from Raman spectroscopy. The FESEM images indicate the CNS were twisted hollowlike structure with irregular shape which may be due to high level of defect mode. The synthesized CNS was deployed directly for sensing without any post treatment and shows a potential application in $\mathrm{NH}_{3}$ gas sensing with an average response time of 2 minutes and recovery time of 5 minutes. This shows that the as grown CNS could be a potential sensing material for ammonia gas sensing.

\section{References}

[1] S. Abdulla, T. L. Mathew, and B. Pullithadathil, "Highly Sensitive Room Temperature Gas Sensor Based On Polyaniline-Multiwalled Carbon Nanotubes (PANI/Mwcnts) Nanocomposite for Trace-Level Ammonia Detection," Sensor and actuators B: Chem, vol. 221, pp. 1523-1534, 2015.

[2] S. Y. Wang et al., "Surface Acoustic Wave Ammonia Sensor Based On $\mathrm{ZnO} / \mathrm{SiO}_{2}$ Composite Film," J. of Hazardous Mat., vol. 238, pp. 368- 374, 2015.

[3] T. Zhang et al., "Recent Progress in Carbon Nanotube-Based Gas Sensor," Nanotech., vol. 19, pp. 1-14, 2008.

[4] L. Zhao et al., "The Effect of Multiwalled Carbon Nanotubes Doping On The CO Gas Sensitivity of $\mathrm{SnO}_{2}$ Based Nanomaterials," Nanotech., vol. 18, pp. 44, 2007.

[5] Z. Yunusa et al., "Synthesis of Y-Tip Graphitic Nanoribbons from Alcohol Catalytic Chemical Vapor Deposition on Piezoelectric Substrate," Nanotech., vol. 2015, pp. 1-8, 2015.

[6] B. Reeti, and D. W. Hanoch, "Fast Growth of 
Carbon Nanotubes Using Microwave Oven," Carbon, vol. 82, pp. 327- 336, 2014.

[7] N. Kure et al., "Synthesis of Carbon Nanotubes Using Microwave Oven," IEEE Explore, 2015.

[8] N. Kure et al., "Simple Microwave-Assisted Synthesis of Carbon Nanotubes Using Polyethylene as Carbon Precursor," J. of Nanomat., Vol. 2017, pp. 1-4, 2017.

[9] T. Charinpanitkul, W. Tanthapanichakoon, and N. Sano, "Carbon Nanostructures Synthesized by Arc Discharge Btween Carbon and Iron Electrodes in Liquid Nitrogen," Curr. Applied Physics, vol. 6, pp. 629-632, 2009.

[10] J. Prasek et al., "Methods for Carbon Nanotubes Synthesis-Review," J. of Material Chem., vol. 21, pp. 15872-15884, 2011.

[11]A. B. Suriani et al., "Synthesis of Vertically Aligned Carbon Nanotubes Using Natural Palm Oil as Carbon Precursor," Mats. Letts., vol. 101, pp. 61-64, 2013.

[12] S. Rahmanian, A. R. Suraya, and R. Zahari, Synthesis of Vertically Aligned Carbon Nanotubes on Carbon Fiber," Appl. Surface Sci., vol. 271, pp. 424-428, 2013.

[13] M. S. Dresselhaus et al., "Raman Spectroscopy on Isolated Single Wall Carbon Nanotubes," Physica B, vol. 323, pp. 15-20, 2002.

[14]L. Bokobza, "Raman Spectroscopic Characterization of Multiwall Carbon Nanotubes and of Composites," Express Polymers Letts., vol. 6, pp. 601-608, 2012. 